\title{
ANÁLISIS MOLECULAR DE LA DIVERSIDAD GENÉTICA EN GUANÁBANA (Annona muricata L.) MEDIANTE MARCADORES SRAP
}

\author{
MOLECULAR ANALYSIS OF GENETIC DIVERSITY IN SOURSOP \\ (Annona muricata L.) USING SRAP MARKERS
}

\section{Carlos A. Talamantes-Sandoval1, ${ }^{1}$, Moisés Cortés-Cruz ${ }^{2}{ }^{,}$Rosendo Balois-Morales ${ }^{3}$, Graciela G. López-Guzmán ${ }^{3}$ y Yolotzin A. Palomino-Hermosillo ${ }^{3}$}

\begin{abstract}
'Universidad Autónoma de Nayarit, Unidad Académica de Agricultura, Xalisco, Nayarit, México. ${ }^{2}$ Instituto Nacional de Investigaciones Forestales, Agrícolas y Pecuarias, Centro Nacional de Recursos Genéticos, Tepatitlán, Jalisco, México. ${ }^{3}$ Universidad Autónoma de Nayarit, Posgrado en Ciencias Biológico Agropecuarias-Unidad de Tecnología de Alimentos, Tepic. Nayarit. México.
\end{abstract}

*Autor de correspondencia (cortes.moises@inifap.gob.mx)

\section{RESUMEN}

El conocimiento sobre la diversidad genética de las plantas cultivadas es de gran importancia ante escenarios de cambio climático y presencia de plagas y enfermedades. México es considerado uno de los países con más diversidad de especies y con una alta producción agrícola; por lo tanto, los cultivos con baja diversidad genética se encuentran en una situación de vulnerabilidad. El estudio de la diversidad genética en plantas puede abordarse mediante dos metodologías: la caracterización morfológica y la molecular. La primera metodología depende de las interacciones con el ambiente, pudiendo generar datos ambiguos, mientras que la segunda, al detectar secuencias específicas en el ADN, se considera estable y de mayor confianza. El objetivo de esta investigación fue evaluar, por primera vez, la capacidad de los marcadores moleculares SRAP (Polimorfismos Amplificados Relacionados con la Secuencia) para determinar el nivel de diversidad genética de 88 genotipos de guanábana (Annona muricata L.) provenientes de cinco huertos comerciales del municipio de Compostela, Nayarit, México (principal productor a nivel mundial). Se seleccionaron tres combinaciones polimórficas y reproducibles de marcadores SRAP que revelaron 126 alelos y $76.67 \%$ de polimorfismo, el número promedio de bandas observadas con estas combinaciones fue de 42 . Los valores de heterocigosidad esperada $\mathrm{He}$ ) variaron entre las poblaciones de 0.144 a 0.176 . Los análisis de agrupamiento mostraron cuatro grupos principales. Los resultados del análisis molecular de varianza indicaron que la mayor variación se encuentra dentro de las poblaciones (88 \%). La información generada de esta investigación puede ser útil para construir estrategias de conservación y manejo del cultivo.

Palabras clave: Annona muricata, diversidad genética, marcadores moleculares SRAP, polimorfismo.

\section{SUMMARY}

Knowledge of genetic diversity of cultivated plants is important under the climate change scenario and the presence of pests and diseases. Mexico is considered one of the countries with the largest diversity of species and agricultural production; therefore, crops with low genetic diversity are in a vulnerable situation. The study of genetic diversity in plants can be addressed through two methodologies: morphological and molecular characterization. The first methodology depends on environmental interactions, generating ambiguous data, while the second one, being able to detect specific DNA sequences, is considered stable and more reliable. The objective of this research was to evaluate, for the first time, the ability of SRAP (Sequencerelated amplified polymorphisms) molecular markers to determine the levels of genetic diversity of 88 soursop (Annona muricata L.) genotypes from five commercial orchards of the municipality of Compostela, Nayarit, Mexico (the world's leading producer). Three polymorphic and reproducible combinations of SRAP markers were selected that revealed 126 alleles and $76.67 \%$ polymorphism, the average number of bands observed with these combinations was 42 . The expected heterozygosity $(\mathrm{He})$ values varied among populations from of 0.144 to 0.176 . Cluster analyses showed four main groups. Results of the analysis of molecular variance indicated that the largest variation $(88 \%)$ is found within populations. The information generated from this research can be useful to devise strategies for conservation and crop management.

Index words: Annona muricata, genetic diversity, SRAP molecular markers, polymorphism

\section{INTRODUCCIÓN}

La familia Annonaceae incluye 130 géneros y 3200 especies, de los cuales el género Annona destaca por su importancia económica y ecológica (Kelly, 2000). La guanábana (Annona muricata L.) es un cultivo de importancia económica del cual México ocupa el primer lugar a nivel mundial, con una producción anual de más de 24 mil t y un valor de la producción de 160 millones de pesos. El estado de Nayarit es el principal productor de este fruto, con el $73 \%$ de la producción nacional, y es en los municipios de Compostela, San Blas, Tepic y Bahía de Banderas donde se concentra la mayor producción (SIAP, 2017).

El centro de origen de esta especie ha sido un tema de controversia entre los expertos; sin embargo, diferentes autores mencionan a las Indias Occidentales como la región más probable donde se originó la especie (De Candolle, 2011; Pinto et al., 2005). Los registros arqueológicos indican la presencia de esta especie desde tiempos prehispánicos, desde Centro América hasta el sur de Perú (Pozorski y Pozorski, 1997).

La propagación de las anonas es usualmente por semilla, 
con excepción de chirimoya (A. cherimola Mill.) y atemoya (A. cherimola $\times$ A. squamosa) (George y Nissen, 1987). Los frutos de guanábana en México presentan una gran diversidad debido a que la mayoría de las plantaciones está constituida por árboles propagados por semilla (Evangelista-Lozano et al., 2003).

La medición de la diversidad genética, que puede realizarse morfológica y molecularmente, es un medio para clasificar e identificar rasgos potencialmente valiosos de los cultivos, así como seleccionar variedades locales que podrían ser utilizadas en programas de mejoramiento genético. El desarrollo y aplicación de las herramientas moleculares en este campo ha sobrepasado las limitantes de la caracterización morfológica; sin embargo, para su selección y aplicación deben considerarse varios factores como su disponibilidad, facilidad, costos de implementación, cobertura del genoma, transferibilidad y cantidad de datos generados por los mismos (Anuragi et al., 2016).

Existen numerosos sistemas de marcadores moleculares que pueden ser empleados, dependiendo de los objetivos del estudio; generalmente, aquellos basados en la reacción en cadena de la polimerasa (PCR) son preferidos sobre otros sistemas que emplean enzimas o procesos de hibridación. Un ejemplo de estos marcadores son los polimorfismos amplificados relacionados con la secuencia o SRAP, por sus siglas en inglés. Estos marcadores son de naturaleza dominante, de fácil implementación en laboratorio y altamente polimórficos, ya que con pocas combinaciones de cebadores se obtienen numerosas bandas; además, son marcadores reproducibles, de aplicación versátil y para su implementación no se requiere de conocimiento previo del genoma de la especie en estudio (Li y Quiros, 2001).

Estos marcadores se basan en la amplificación de ADN mediante dos cebadores de 17 ó 18 nucleótidos de longitud. Cada cebador posee una secuencia núcleo de 13 a 14 pb de longitud. Los primeros 10 u 11 nucleótidos del extremo 5' son secuencias sin constitución específica (secuencias de "relleno"); después de estos 10 u 11 nucleótidos continua una secuencia de anclaje que es diferente para cada cebador: "CCGG" en el cebador directo y "AATT" en el cebador inverso. Estas secuencias de anclaje están dirigidas hacia regiones codificantes del genoma, permitiendo la detección de polimorfismos; finalmente, la secuencia del cebador se completa en el extremo 3' con tres nucleótidos selectivos (Li y Quiros, 2001).

Actualmente, existe una creciente preocupación sobre la pérdida acelerada de la diversidad genética en los cultivos. Esto ha llevado a tomar acciones para la conservación de los recursos genéticos, ya sea bajo esquemas de conservación in situ o ex situ a través de bancos de germoplasma; sin embargo, en el caso de frutales tropicales, como la guanábana, existen vacíos de información sobre la distribución de la diversidad genética. El objetivo del presente estudio fue evaluar la efectividad de los marcadores moleculares SRAP para determinar los valores de diversidad genética en 88 genotipos de guanábana (A. muricata L.) provenientes de cinco huertos comerciales del municipio de Compostela, Nayarit, México.

\section{MATERIALES Y MÉTODOS}

\section{Material vegetal}

Tejido foliar joven de árboles de guanábana en producción, libre de daños físicos, mecánicos y microorganismos fue recolectado en cinco huertos comerciales ubicados en las localidades La Puerta de la Lima, Chacala y El Tonino, del municipio de Compostela, Nayarit, México. El material vegetal fue almacenado a $-80{ }^{\circ} \mathrm{C}$ en un ultra congelador (Haier Bio Medical, Qigdao, China) por un periodo mínimo de 48 h. Después se liofilizó en un aparato FreeZone 2.5 (Labconco, Kansas City, MO, USA) a una temperatura de $-85^{\circ} \mathrm{C}$ y presión de $0.040 \mathrm{mBar}$ durante 7 días, para posteriormente ser pulverizados en un molino eléctrico (KRUPS, Solingen, Alemania), previo a la extracción de ADN.

\section{Extracción de ADN}

El ADN genómico total fue extraído de acuerdo con la metodología de Saghai-Maroof et al. (1984), con algunas modificaciones: en tubos de $2 \mathrm{~mL}$ se colocaron $50 \mathrm{mg}$ de tejido liofilizado y pulverizado al cual se agregó $1 \mathrm{~mL}$ de Buffer de extracción ( $\mathrm{ddH}_{2} \mathrm{O}, 100 \mathrm{mM}$ tris HCL-7.5, $700 \mathrm{mM}$ $\mathrm{NaCl}, 50$ mM EDTA-8.0, CTAB $3 \%$, 320 mM BME y PVP $3 \%$ ), previamente calentado $65^{\circ} \mathrm{C}$, mezclándolo perfectamente con el tejido. Las muestras fueron incubadas a $65^{\circ} \mathrm{C}$ durante 60 min en agitación constante y suave; transcurrido este tiempo, las muestras se dejaron enfriar aproximadamente por 5 min y se adicionaron $700 \mu \mathrm{L}$ de cloroformo:octanol $(24: 1 \mathrm{v} / \mathrm{v})$ mezclando suavemente por inversión durante 15 min, seguido de un paso de centrifugación a 14,000 rpm en una centrífuga de mesa (Thermo Scientific, Waltham, MA, USA) a temperatura ambiente. El sobrenadante fue transferido a un tubo nuevo de $2 \mathrm{~mL}$, repitiendo el proceso. El sobrenadante se transfirió a un tubo nuevo de $2 \mathrm{~mL}$ que contenía $10 \mu \mathrm{L}$ de RNasa (10 $\left.\mathrm{mg} \mathrm{mL}^{-1}\right)$, incubándose las muestras durante $30 \mathrm{~min}$ a $37^{\circ} \mathrm{C}$. Después de este tiempo, el ADN se precipitó con $800 \mu \mathrm{L}$ de etanol frio $\left(-20^{\circ} \mathrm{C}\right)$ invirtiendo con suavidad los tubos hasta lograr la formación de la pastilla de ADN, para después centrifugar a 10,000 rpm durante 6 min y decantar el sobrenadante, cuidando de que la pastilla de ADN no se desprendiera 
del fondo. Se realizaron dos lavados con etanol al 75 y 95 $\%$. En cada lavado se adicionaron $1000 \mu \mathrm{L}$ de la solución, mezclando suavemente los tubos por inversión durante 10 min para después centrifugar a 10,000 rpm durante 10 min. Después de la segunda centrifugación, las pastillas de ADN se dejaron secar para evaporar completamente el alcohol y después fueron disueltas en $300 \mu \mathrm{L}$ de buffer TE (10mM Tris-HCl pH 8, 1 mM EDTA). Las muestras disueltas fueron almacenadas a $-20^{\circ} \mathrm{C}$ hasta su utilización.

\section{Cuantificación del ADN}

La cantidad y calidad del ADN fue determinada mediante espectrofotometría (260/280 OD) en un equipo Nanodrop 2000 (Thermo Scientific, Waltham, MA, USA); además, se realizó una inspección visual de las muestras mediante electroforesis de $100 \mathrm{ng}$ de ADN genómico total en geles de agarosa 1 \% y los perfiles de migración de las muestras fueron comparados con el marcador de referencia Lambda sin cortar.

\section{Análisis SRAP}

Se evaluaron 40 combinaciones de marcadores SRAP (4 directos y 10 inversos), de las cuales 18 resultaron polimórficas, seleccionándose finalmente tres combinaciones cuyos patrones de amplificación fueron reproducibles y con el mayor número de bandas (Cuadro 1).

Las reacciones de amplificación se realizaron en un volumen final de $10 \mu \mathrm{L}$ que contenía $0.8 \mathrm{X}$ de REDTaq ${ }^{\circledR}$ ReadyMix ${ }^{\text {TM }}$ PCR Reaction Mix (Sigma-Aldrich, St. Louis, MO, USA), $0.5 \mathrm{M}$ de cada cebador, $12.5 \mathrm{ng}$ de ADN y agua bidestilada estéril. La amplificación se realizó en un termociclador Veriti 96 Well Thermal Cycler (Applied Biosystems, Foster City, CA, USA). Las condiciones de amplificación incluyeron una etapa de desnaturalización inicial a $94^{\circ} \mathrm{C}$ durante $5 \mathrm{~min}$, seguida por dos fases de amplificación. La primera fase consistió de cinco ciclos, desnaturalización a $94{ }^{\circ} \mathrm{C}$ por 1 min, alineamiento a $35^{\circ} \mathrm{C}$ por 1 min y elongación a $72^{\circ} \mathrm{C}$ por 1 min; la segunda fue de 35 ciclos donde solamente se incrementó la temperatura de alineamiento a $50^{\circ} \mathrm{C}$. El paso de elongación final fue de $72{ }^{\circ} \mathrm{C}$ durante $10 \mathrm{~min}$.

Los productos de PCR fueron separados mediante electroforesis vertical en geles de poliacrilamida $8 \%$. El corrimiento electroforético se realizó en las cámaras SG-160 (CBS Scientific, San Diego, CA, USA) a 250V por 2 h. La visualización de los fragmentos amplificados se realizó siguiendo la metodología de tinción con nitrato de plata (Sanguinetti et al., 1994) y se fotografiaron bajo luz blanca con el equipo CareStream Gel Logic 100 (Kodak, CareStream Health, Rochester, NY, USA).

\section{Análisis de la información}

Con los perfiles obtenidos a partir de los geles de poliacrilamida se construyó una matriz binaria de datos dominantes ( 1 para presencia y 0 para ausencia); se seleccionaron solamente los fragmentos que se ubicaron en el intervalo de 100 a 500 pb. Los datos fueron analizados con el software GenAlEx 6.5 (Peakall y Smouse, 2012) para calcular parámetros de diversidad genética a nivel de población como porcentaje de loci polimórficos $(\% \mathrm{P})$ y heterocigosidad esperada $(\mathrm{He})$ y realizar análisis de varianza molecular (AMOVA). Con base en la matriz de distancias genéticas se construyó un dendrograma basado en el método de Neighbor-Joining (Nei, 1977) utilizando el programa MEGA 5 (Tamura et al., 2011).

Se realizó una prueba de estructura genética mediante un análisis Bayesiano de asignación de individuos en el cual de una a cinco poblaciones podían estar agrupadas. El programa fue ejecutado usando 250,000 iteraciones de MCMC (cadenas de Markov Monte Carlo), 50,000 réplicas y 10 repeticiones para cada $K$, empleando el programa de STRUCTURE 2.3.4. (Evanno et al., 2005).

Cuadro 1. Nombre y secuencia de las tres combinaciones de cebadores SRAP utilizados en el análisis de 88 genotipos de guanábana (Annona muricata L.).

\begin{tabular}{lllll}
\hline Cebador & Secuencia $\left(5^{\prime}-3^{\prime}\right)$ & Cebador & Secuencia $\left(3^{\prime}-5^{\prime}\right)$ & NB $^{+}$ \\
\hline me3 & TGAGTCCAAACCGGAAT & em1 & GACTGCGTACGAATTAAT & 41 \\
me3 & TGAGTCCAAACCGGAAT & em15 & GACTGCGTACGAATTGAT & 44 \\
me4 & TGAGTCCAAACCGGACC & em15 & GACTGCGTACGAATTGAT & 41 \\
\hline
\end{tabular}

${ }^{\dagger} \mathrm{NB}$ : número de bandas observadas en este estudio por combinación de cebadores. 


\section{RESULTADOS Y DISCUSIÓN}

Se reporta por primera vez el uso de marcadores SRAP en una especie del género Annona. Las tres combinaciones de cebadores fueron exitosas, amplificando un total de 126 alelos en el análisis de los 88 genotipos. El valor promedio de polimorfismo fue $76.67 \%$, con una amplitud de 83.33 \% (La Puerta de la Lima 2) a 69.84 \% (El Tonino). El valor promedio de \% $P$ fue mayor en este estudio que los valores reportados para otras especies del género Annona. Bharad et al. (2009) y Guimaraes et al. (2013), empleando marcadores RAPD en A. squamosa, reportaron valores de polimorfismo de 73.7 y $29 \%$ respectivamente. Estos valores sugieren un mayor poder de detección de polimorfismo de los marcadores SRAP en comparación con los marcadores RAPD.

Como parte de la selección de marcadores para la realización del presente estudio se evaluó la transferibilidad de marcadores microsatélites de A. chirimoya en $A$. muricata. Se seleccionaron 23 marcadores con base en el valor del contenido de información polimórfica (PIC) (Escribano et al., 2008). La amplificación de 13 marcadores fue exitosa, mientras que en el resto de los marcadores evaluados no hubo amplificación; sin embargo, todos los marcadores que amplificaron fueron monomórficos (datos no presentados). Estos resultados confirman la aplicabilidad y versatilidad de los marcadores SRAP, donde no es necesario tener conocimiento previo del genoma de la especie en estudio para su implementación.

El valor de la He fue variable a través de las cinco poblaciones. El valor promedio fue de $\mathrm{He}=0.164$, con una fluctuación de $\mathrm{He}=0.175$ para la población de La Puerta de la Lima 2, hasta $\mathrm{He}=0.144$ para la población de Chacala 2. El valor promedio de He obtenido en el presente estudio fue bajo en comparación con los valores reportados por otros autores en diferentes especies del mismo género. Escribano et al. (2007) evaluaron 279 accesiones de chirimoya (A. cherimola) con 16 marcadores SSR y reportaron un valor promedio de $\mathrm{He}=$ 0.50 . Un valor de $\mathrm{He}=0.57$ fue reportado por Escribano et al. (2008) al evaluar 52 SSR en 23 accesiones de chirimoya de diversas áreas geográficas. Por otra parte, Salazar et al. (2010), analizando 14 poblaciones de A. squamosa de la Península de Yucatán, reportaron un valor promedio de $\mathrm{He}=0.47$ mediante el empleo de isoenzimas. Estos valores deben ser considerados con cuidado, por haber empleado diferentes sistemas de marcadores y por la naturaleza de la variación de cada uno de ellos, además del origen genético de los materiales evaluados; sin embargo, pueden ser una referencia de valores de diversidad, ya que no existen reportes en la literatura sobre la distribución de la diversidad genética de guanábana en México.

El análisis de varianza molecular (AMOVA) se realizó considerando las cinco poblaciones. El mayor porcentaje de variación se detectó dentro de poblaciones (88\%), en contraste con la variación entre poblaciones (12\%). Considerando que el sistema tradicional de propagación de la especie es a través de semillas, este valor refleja la variabilidad que existe dentro de cada población y su grado de aislamiento.

Actualmente existe una tendencia a seleccionar en campo individuos que sean similares en diferentes caracteres; por ejemplo, en el número de días a floración o fructificación. Al buscar la sincronización de la floración y fructificación se facilitan algunas tareas de campo, sobre todo al momento de la cosecha; sin embargo, esta selección conlleva hacia una reducción de la diversidad de la especie, como lo sugieren los resultados del presente estudio. Esta baja diversidad pone en riesgo la sobrevivencia de la especie ante escenarios de cambio climático, o de aparición de nuevas plagas y enfermedades en esta importante zona de producción de guanábana.

El análisis bayesiano de asignación de individuos mostró que el número más probable de grupos genéticos es $K=4$ (Figura 1A). En este escenario, se asumió un modelo de entrecruzamiento y que las poblaciones derivan de un ancestro

Cuadro 2. Parámetros básicos de diversidad genética de 88 genotipos de anona (Annona muricata L.) de cinco localidades de Nayarit, México.

\begin{tabular}{llccl}
\hline Población & $\mathrm{N}^{+}$ & No. de loci polimórficos & \% de polimorfismo & $\mathrm{He}^{\dagger \dagger}$ \\
\hline La Puerta de la Lima 1 & 20 & 95 & 75.40 & 0.175 \\
La Puerta de la Lima 2 & 20 & 105 & 83.33 & 0.167 \\
Chacala 1 & 14 & 96 & 76.19 & 0.176 \\
Chacala 2 & 20 & 99 & 78.57 & 0.144 \\
El Tonino & 14 & 88 & 69.84 & 0.157 \\
Promedio & & 94.8 & 76.67 & 0.164 \\
\hline
\end{tabular}

${ }^{\top}$ Número de individuos por población, ${ }^{+} H e$ : heterocigosidad esperada. 
común. Los análisis estadísticos indicaron que solamente la población de Chacala 2 presentó un valor de coeficiente de coancestría $\geq 80 \%$ mientras que el resto de las poblaciones presentaron valores menores, indicando una mezcla de grupos genéticos dentro de cada población (Figura 1B, Cuadro 3).

El dendrograma dividió a los 88 genotipos en cuatro clados principales, corroborando los resultados del análisis de STRUCTURE (Figura 1c). Los clados II y IV contienen en su mayoría a los individuos de las poblaciones Chacala 2 y La puerta de Lima 1, respectivamente. Estos agrupamientos corresponden con los valores más altos del coeficiente de coancestría (Cuadro 3). Los clados I y III agrupan individuos de diferentes poblaciones, corroborando la variabilidad que existe dentro de poblaciones.

El conocimiento de la diversidad y estructura genética en guanábana es esencial para identificar los riesgos actuales y elucidar algunas medidas de protección para esta especie. Los resultados de este estudio muestran niveles bajos de diversidad genética en comparación con otras especies del mismo género. Con el fin de revertir esta situación y conservar e incrementar los niveles de diversidad genética se pueden diseñar diferentes estrategias. Una de las primeras estrategias prioritarias sería la identificación de

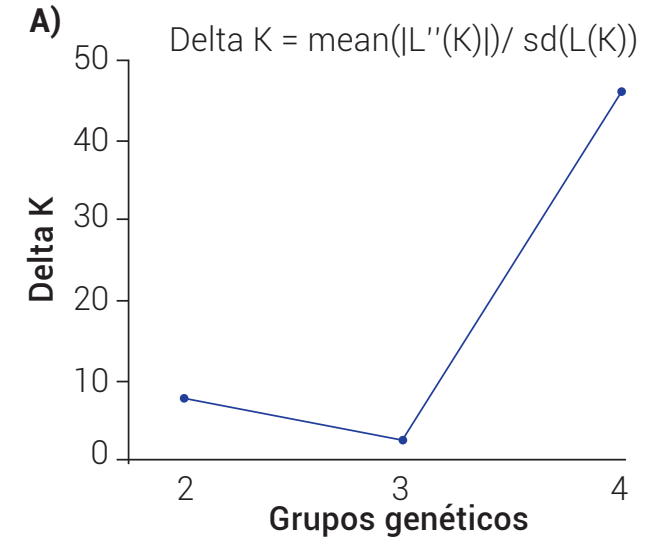

- La Puerta de la Lima 1

+ La Puerta de la Lima 2

- Chacala 1

- Chacala 2

- El tonino

genotipos individuales portadores de riqueza alélica con fines de conservación. La conservación in situ de estos materiales deberá ser complementada con conservación ex situ; sin embargo, debido a la naturaleza recalcitrante de las semillas, se deben visualizar alternativas como la conservación in vitro bajo condiciones mínimas de crecimiento o criopreservación. Adicionalmente, el intercambio de semillas entre productores de la región puede favorecer la creación de nuevas combinaciones alélicas debido a la recombinación genética durante el proceso de formación de gametos. Estas actividades podrían incrementar a largo plazo el acervo genético de las plantaciones y de esta manera disminuir la vulnerabilidad de las mismas ante las amenazas ambientales actuales.

\section{CONCLUSIONES}

Este es el primer reporte sobre diversidad genética empleando marcadores moleculares SRAP en guanábana, demostrando la utilidad y confiabilidad de estos marcadores para la estimación de los parámetros de diversidad y las relaciones entre los genotipos en las poblaciones de esta especie. Los niveles de diversidad genética que se obtuvieron en el municipio de Compostela, Nayarit, México fueron de bajos a moderados en comparación con lo

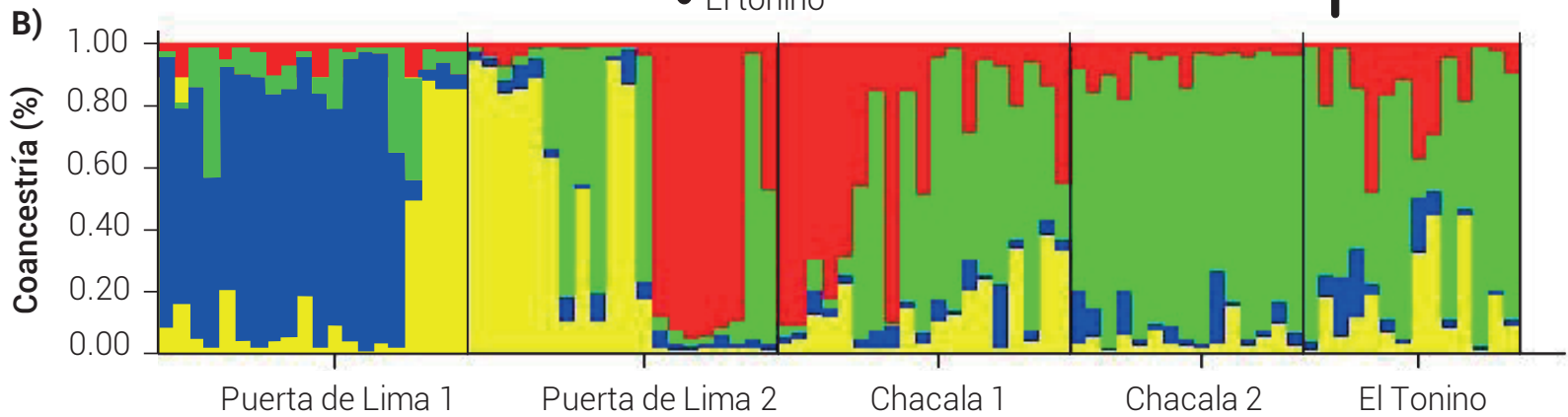

Figura 1. A) Valores de Delta $K$ para el análisis de STRUCTURE calculado según Evanno et al. (2005), graficados contra el número de grupos genéticos $(K)$; B) representación gráfica de la estructura genética de las cinco poblaciones de guanábana (Annona muricata L.) donde los grupos genéticos ( $K$ ) están indicados por los colores sobre el eje $\mathrm{X} ; \mathrm{C}$ ) dendrograma de 88 genotipos de guanábana con base en el método Neigbor-Joining, los cuatro clados principales están identificados por los círculos sombreados. 
Cuadro 3. Valores de coancestría ( $Q$ ) estimados para las cinco poblaciones estudiadad de guanábana (Annona muricata L.).

\begin{tabular}{lcccc}
\hline \multirow{2}{*}{ Población } & \multicolumn{4}{c}{ Grupo Genético } \\
\cline { 2 - 5 } & I & II & III & IV \\
\hline Puerta de Lima 1 & 0.046 & 0.101 & 0.646 & 0.208 \\
Puerta de Lima 2 & 0.317 & 0.243 & 0.043 & 0.397 \\
Chacala 1 & 0.419 & 0.396 & 0.051 & 0.134 \\
Chacala 2 & 0.069 & 0.820 & 0.064 & 0.047 \\
El Tonino & 0.156 & 0.615 & 0.069 & 0.160 \\
\hline
\end{tabular}

reportado en otros trabajos realizados en especies de la familia Annonaceae, lo que indica la vulnerabilidad de las plantaciones comerciales a factores adversos en las localidades analizadas. Con base en los resultados del presente estudio, para incrementar la diversidad en los huertos comerciales establecidos es necesario diseñar estrategias de conservación y propagación sexual para promover la recombinación genética y explorar la posibilidad de generación de nuevos materiales para incrementar el acervo genético de las poblaciones.

\section{AGRADECIMIENTOS}

Esta investigación fue financiada por el Fondo Sectorial de Investigación para la Educación establecido por la Secretaría de Educación Pública y el Consejo Nacional de Ciencia y Tecnología a través del Proyecto No. 242718: "Caracterización morfológica, bioquímica y genética de guanábana (Annona muricata L.)".

\section{BIBLIOGRAFÍA}

Anuragi H., H. L. Dhaduk, S. Kumar, J. J. Dhruve, M. J. Parekh and A. A. Sakure (2016) Molecular diversity of Annona species and proximate fruit composition of selected genotypes. 3 Biotech 6:204, https://doi.org/10.1007/s13205-016-0520-9

Bharad S. G., P. L. Kulwal and S. A. Bagal (2009) Genetic diversity study in Annona squamosa by morphological, biochemical and RAPD markers. Acta Horticulturae 839:615-623, https://doi.org/10.17660/ActaHortic.2009.839.84

De Candolle A. (2011) Origin of Cultivated Plants. Cambridge University Press. New York. 468 p. https://doi.org/10.1017/CB09781139107365

Escribano P., M. A. Viruel and J. I. Hormaza (2007) Molecular analysis of genetic diversity and geographic origin within an ex situ germplasm collection of cherimoya by using SSRs. Journal of the American Society for Horticultural Science 132:357-367, https://doi.org/10.21273/JASHS.132.3.357

Escribano P., M. A. Viruel and J. I. Hormaza (2008) Development of 52 new polymorphic SSR markers from cherimoya (Annona cherimola Mill.): transferability to related taxa and selection of a reduced set for DNA fingerprinting and diversity studies. Molecular Ecology Resources 8:317-321, https://doi.org/10.1111/j.1471-8286.2007.01941.x

Evangelista-Lozano S., J. G. Cruz-Castillo, S. Pérez-González, E. MercadoSilva y G. Dávila-Ortiz (2003) Producción y calidad frutícola de guanábanos (Annona muricata L.) provenientes de semilla de Jiutepec, Morelos, México. Revista Chapingo Serie Horticultura 9:69-79.

Evanno G., S. Regnaut and J. Goudet (2005) Detecting the number of clusters of individuals using the software STRUCTURE: a simulation study. Molecular Ecology 14: 2611-2620, https://doi.org/10.1111/j.1365-294X.2005.02553.x

George A. P. and R. J. Nissen (1987) The effects of day/night temperatures on growth and dry matter production of custard apple (Annona cherimola $\times$ Annona squamosa) cultivar 'African Pride'. Scientia Horticulturae 31: 269-274, https://doi.org/10.1016/0304-4238(87)90052-5

Guimaraes R. J. F., S. Nietsche y G. B. Rocha (2013) Diversidad genética en la manzana de azúcar (A. squamosa L.) utilizando marcadores RAPD. Revista Ceres 60:428-431.

Kelly L. M. (2000) Annonaceae Juss. In: Flora del Valle de TehuacánCuicatlán. Vol. 31. L. M. Kelly, H. Ochoterena y R. Medina L. (eds.). Instituto de Biología, Universidad Nacional Autónoma de México. México, D. F. pp:1-5.

LiG. and C. F. Quiros (2001) Sequence-related amplified polymorphism (SRAP), a new marker system based on a simple PCR reaction: its application to mapping and gene tagging in Brassica. Theoretical and Applied Genetics 103:455-461, https://doi.org/10.1007/s001220100570

Nei M. (1977) F-statistics and analysis of gene diversity in subdivided populations. Annals of Human Genetics 41:225233, https://doi.org/10.1111/j.1469-1809.1977.tb01918.x

Peakall R. andP.E. Smouse(2012) GenAlEx 6.5: genetic analysis in Excel. Population genetic software for teaching and research-an update. Bioinformatics 28:2537-2539, https://doi.org/10.1093/bioinformatics/bts460

Pinto A. C. Q., M. C. R Cordeiro, S. R. M. De Andrade, F. R. Ferreira, H. A. C. Filgueiras, R. E. Alves and D. I. Kinpara (2005) Annona species. International Centre for Underutilised Crops. University of Southampton. Southampton, UK. 268 p.

Pozorski T. and S. Pozorski (1997) Cherimoya and guanabana in the archaeological record of Peru. Journal of Ethnobiology 17:235248.

Saghai-Maroof M. A., K. M. Soliman, R. A. Jorgensen and R. W. Allard (1984) Ribosomal DNA spacer-legth polymorphisms in barley: Mendelian inheritance, chromosomal location, and population dynamics. Proceeding of the National Academy of Science of the United States of America 81:8014-8018, https://doi.org/10.1073/pnas.81.24.8014

Salazar C., C. F. Vargas-Mendoza y J. Salvador F. (2010) Estructura y diversidad genética de Annona squamosa en huertos familiares mayas de la península de Yucatán. Revista Mexicana de Biodiversidad 81: 759-770.

Sanguinetti C. J., E. D. Neto and A. J. G. Simpson (1994) Rapid silver staining and recovery of PCR product separated on polyacrylamide gels. BioTechniques 17:914-921.

SIAP, Servicio de Información Agroalimentaria y Pesquera (2017) Anuario estadístico de la producción agrícola. Servicio de Información Agroalimentaria y Pesquera, Secretaría de Agricultura, Ganadería, Desarrollo Rural, Pesca y Alimentación. México, D. F. http://nube.siap.gob.mx/cierre_agricola/ (Agosto 2017).

Tamura K., D. Peterson, N. Peterson, G. Stecher, M. Nei and S. Kumar (2011) MEGA 5: Molecular evolutionary genetics analysis using maximum likelihood, evolutionary distance and maximum parsimony methods. Molecular Biology and Evolution 28:27312739, https://doi.org/10.1093/molbev/msr121 